\title{
Rotational Auto-detachment of Dipole-Bound Anions
}

\author{
S. G. Ard ${ }^{1,2}$, R.N. ,Compton ${ }^{1,3}$ and W.R. Garrett ${ }^{1}$ \\ 1. Department of Physics, The University of Tennessee, Knoxville, Tennessee 37996 \\ 2. Air Force Research Lab, Space Vehicles Directorate, Kirtland AFB, New Mexico 87117 \\ 3. Department of Chemistry, The University of Tennessee, Knoxville, Tennessee 37996
}

\begin{abstract}
Rotational auto-detachment of of acetonitrile, trimethyl-acetonitrile, acetone, and cyclobutanone dipole-bound anions was studied under varying conditions in a Rydberg electron transfer (RET) time-of-flight apparatus. Varying amounts of auto-detatchment was observed for anions with similar electron affinity and dipole moment, but different moments of inertia. These results were found to be consistent with predictions based on the calculated rotational spectra for these anions, highlighting the importance of critical binding properties in understanding the stability and lifetime of dipole bound systems.
\end{abstract}

\section{Introduction}

An electron can be bound to any molecule that possesses a sufficiently large dipole moment into what is often called a dipole-bound negative ion state. Numerous authors ${ }^{1-9}$ have calculated the minimum dipole moment of 1.625 Debye for electron binding to a fixed dipole. Below this critical value, no bound states are supported by the electric dipolar field, and for dipole moments above this value an infinite number of bound states exist. However, the critical moment for binding to a "real" molecule depends upon the dipole length, the moment of inertia, and the rotational quantum state of the finite dipole $^{10}$. These calculations show a low binding energy as a function of the dipole moment for linear molecules until approximately 2 Debye where the binding energy becomes comparable to the rotational energy of most molecules. This prompted Crawford and Garrett ${ }^{11}$ to predict that any polar molecule with a dipole moment above 2 Debye will form a bound state. Although electron binding to molecules with dipole moments as low as 2 Debye have not yet been reported, these general predictions have been verified for a large number of polar molecules (see the reviews by Desfrancois et al. ${ }^{12}$ and Compton and Hammer ${ }^{13}$ ). Electron affinities for many of these molecules are reported in these reviews using Rydberg Electron Transfer (RET), field detachment, and photoelectron spectroscopy methods. In one study, dipole-bound electron affinities for 40 polar molecules have been published and the electron affinities range from $\sim 0.5 \mathrm{meV}$ for low ( 2.5 Debye) dipole moment molecules to $\sim 20 \mathrm{meV}$ for high ( $\sim 4$ Debye) dipole moment molecules. ${ }^{14}$ In these studies it was necessary to employ nozzle-jet expansion of the polar molecules with low dipole moments in order to produce stable anions. From these observations it has been assumed that at room temperature molecules of the weakly polar species are subject to rapid rotational auto-detachment. The stability of these weakly bound, diffuse dipole-bound anions is also sensitive to electric fields and collisions. Seuss et al. ${ }^{15,16}$ have also shown that dipole-bound anions held in a Penning trap possess relatively short lifetimes ( $\sim 50$ to $100 \mu \mathrm{sec})$ which was attributed to black- 
body-induced photo-detachment. A $300 \mathrm{~K}$ blackbody spectrum peaks at a photon energy of $\sim 120 \mathrm{meV}$, well above the photo-detachment threshold for all known dipole-bound anions. Calculated lifetimes by Chernovet al. ${ }^{17}$ based upon direct photo-detachment are in agreement with these measurements. It is important to note that the stored ions in the experiments by Suess et al., ${ }^{15,16}$ were first made from RET with room temperature gas molecules before injection into the Penning ion trap.

For dipole-bound anions, rotational excitation is very important in its effect on the bound state spectrum and on the lifetimes of such systems. When rotational degrees of freedom are included in non-Born-Oppenheimer calculations of model systems ${ }^{18}$ and real anions, $19,20,21$ the number of excited states of supercritical systems is reduced from an infinite number down to only a few. The rotational spectrum crosses from bound states over to a rich spectrum of rotational resonances of different widths. ${ }^{21,22}$ Clary $^{23}$ and Simons ${ }^{24}$ have considered these effects. Simons made modifications to the original rotationally adiabatic model of Clary to calculate the rotational auto-detachment lifetimes for the $\mathrm{CH}_{2} \mathrm{CN}^{-}$ anion for $\mathrm{J}=31$ to 39 . In the more recent work by Fossez, ${ }^{21}$ the use of complex-energy bases provided bound states and rotational-resonance energies and widths for HCN anions. The groups of Brauman ${ }^{25}$ and Lineberger ${ }^{26}$ have reported very narrow resonance features in the photo-detachment thresholds for valence to dipole-bound anion transitions corresponding to rotationally excited shape and Feshbach resonances for many dipolebound radical anions.

In a recent study, Garrett ${ }^{27}$ has extended results for the polar anion problem to include critical binding by polar symmetric top molecules. For the symmetric top, the effects due to rotational degrees of freedom are very different from the earlier results for linear rotors, ${ }^{18-21}$ indeed a new set of critical moments can be defined for these systems. For symmetric tops possessing non-zero rotations about the axis of symmetry (which contains the direction of the dipolar vector) a finite bound state spectrum transitions into an infinite number of bound states when the dipole moment exceeds newly defined critical values. ${ }^{27}$ These new properties are germane to the present study.

In this study we experimentally examine the auto-detachment lifetimes for a series of dipole-bound anions formed by RET to nozzle expanded polar molecules. The molecules studied include symmetric and asymmetric tops that were chosen for their dipole moments, and moments of inertia.

\section{Experimental}

The apparatus and procedure employed to prepare the dipole-bound anions has been previously described. ${ }^{15-17}$ Briefly, Rydberg electron charge exchange (RET) occurs between the dipolar molecule of interest and highly excited $\mathrm{Rb}$ atoms. The $\mathrm{Rb}$ source consists of a resistively heated alkali oven held at $180^{\circ} \mathrm{C}$ producing a steady vapor of atomic $R b$. This $R b$ vapor was then excited to various $n s^{2} S_{1 / 2}$ and $n d^{2} D_{5 / 2,3 / 2}$ states via resonant two-photon excitation. The states were excited using the output of a tunable pulsed dye laser (Quanta Ray) pumped by the second harmonic $(532 \mathrm{~nm})$ of the fundamental (1064 nm) of an ND:YAG laser (Continuum Powerlite). 
The molecules studied were introduced to the interaction region by an RM Jordan pulsed supersonic valve aligned mutually orthogonal to the beam of excited $\mathrm{Rb}$ atoms and the direction of the time-of-flight mass spectrometer. The molecules were seeded in Argon to promote rotational and vibrational cooling to allow for the greater production of the dipole bound states. The Rydberg atom transfers its electron to the polar molecule forming a dipole-bound anion. After an interaction time of $\sim 700 \mathrm{~ns}$ the anions were extracted with a voltage pulsed to a $\sim 1 \mathrm{~m}$ long time-of-flight mass spectrometer. The ions, as well as neutrals resulting from all subsequent detachment processes (auto-, collisional- and photo-detachment), were then detected using a z-stack channel plate detector. The z-stack was used to more effectively detect low energy neutral particles.

An important aspect of the time-of-flight setup that allows for the detection of the neutrals is a $\sim 10 \mathrm{~cm}$ region prior to the detector that focusses and decelerates the ions. The dipole bound anions will become neutralized as they travel down the flight tube as a result of blackbody photo-detachment and rotational auto-detachment. The neutrals that have been produced will then proceed without this deceleration, allowing for a shorter time of flight enabling them to be distinguished from the ion signal. The neutral signals display a tail to high time to a varying degree, indicating detachment in the decelerating region. The overall time-of-flight as well as the separation in time of the neutrals and ions was measured exactly as predicted from modelling by SIMION $^{28}$.

Verification of the neutral peaks came from the application of both magnetic and electric fields. A movable permanent magnet ( 200 gauss) was placed perpendicular to the path of the ions and neutrals down the flight tube. The ion signal is deflected by this magnetic field and disappears completely while the neutral signal remains unaffected. The magnitude of the neutral signal varied dependent upon how far down the flight tube the magnet was placed. The ability to deflect ions in various positions down the flight tube allows an approximate indication of where the auto-detachment was taking place as well as to evaluate the relative contribution of the blackbody photo-detachment. Additionally, electrodes between the flight tube and detector were held at varying voltages having a pronounced effect on the time-of-flight of the ion signal and not the neutral signal. Finally, stable anion signals, such as $\mathrm{Cl}^{-}$, result in no observable neutral signal despite significantly larger anion signal.

Table 1. Relevant experimental ${ }^{14}$ and calculated structural properties of the molecules studied.

\begin{tabular}{|l|l|l|l|l|}
\hline & Acetonitrile & $\begin{array}{l}\text { Trimethyl- } \\
\text { acetonitrile }\end{array}$ & Acetone & Cyclobutanone \\
\hline EA & $19.3 \mathrm{meV}$ & $13.2 \mathrm{meV}$ & $2.5 \mathrm{meV}$ & $1.7 \mathrm{meV}$ \\
\hline$\mu$ & $3.92 \mathrm{D}$ & $3.95 \mathrm{D}$ & $2.88 \mathrm{D}$ & $2.89 \mathrm{D}$ \\
\hline $\mathrm{I}_{\mathrm{xx}}$ & 11.33 a.u. & 397.41 a.u. & 179.04 a.u. & 169.26 a.u. \\
\hline $\mathrm{I}_{\mathrm{yy}}$ & 198.6 a.u. & 658.33 a.u. & 211.86 a.u. & 374.06 a.u. \\
\hline $\mathrm{I}_{\mathrm{zz}}$ & 198.6 a.u. & 658.33 a.u. & 386.59 a.u. & 499.64 a.u. \\
\hline
\end{tabular}


Table 1 shows the molecules studied, their calculated moments of inertia, and experimentally determined dipole moments and electron affinities. ${ }^{14}$ The dipole moments and electron affinities are important as they are expected to play a role in the blackbody photo-detachment of these anions. ${ }^{1}$ The moments of inertia, primarily those orthogonal to the dipole moment, are likewise expected to play an important role in the relative formation initially and later detachment of the present set of anions. The molecules were chosen as pairs with similar dipole moments and electron affinities, differing primarily in moments of inertia. Note that the first pair are symmetric top molecules, the second pair are asymmetric tops. These sets of molecules were chosen specifically to explore the effects of rotational auto-detachment on the formation and survival of dipole bound anions under various experimental conditions.

Production of the neutral signal was highly dependant on experimental conditions, primarily the background pressure inside the interaction region. This background pressure is affected by the amount of gas pulsed in as well as the pumping taking place in the interaction region, thus exact repetition of experimental conditions were quite difficult. This is rather vital as higher pressures in the interaction region caused a greater relative amount of neutral as compared to the ion signal. This is likely caused by rotational excitation caused by collisions with the background gas. Collisional detachment may be present as well, but the neutrals produced in that manner would not have the full acceleration provided by the pulsed voltage and thus would not be observable.

\section{III.Results}

In order to consolidate the data and to compensate for experimental variability between tests, comparable pairs of molecules were studied simultaneously. This was possible due to each pair of molecules having similar RET spectra (i.e. signal versus Rydberg level or $\left.\mathrm{n}^{*}\right)$, due to their similar electron affinities. Due to potentially varied cooling efficiencies and RET cross sections for the molecules, little can be derived from the relative intensities between the ion pairs in the present experiment, thus we focus on the relative ion to neutral signal observed. Fig. 1 shows the ion and neutral signals from production of dipole bound acetonitrile and trimethyl-acetonitrile anions for various pressures in the interaction region. The previously mentioned pressure dependence is clearly evident. As the pressure in the interaction region is increased, we see an increase in the amount of neutrals detected with minimal impact on the ion signal. In this case the pressure was varied by limiting the out pumping of the interaction region. A similar affect was seen by increasing the pressure in the interaction region by raising the backing pressure of the pulser, or by increasing the current applied to the pulsed valve which increases the 
opening of the valve, though these methods also likely affected the effective cooling of the expansion. Acetonitrile consistently produces more neutral relative to ion signal than for the case of trimethyl acetonitrile, independent of the experimental conditions. This can not be accounted for due to the differences in their electron affinities, as acetonitrile has a slightly higher electron affinity. We attribute the larger auto-detachment to the effects of the moments of inertia of the two molecules. The moment of inertia for trimethyl acetonitrile, primarily along the principal axes perpendicular to the dipole moment, is larger than that of acetonitrile. We explore the implications of these factors in theoretical considerations below.

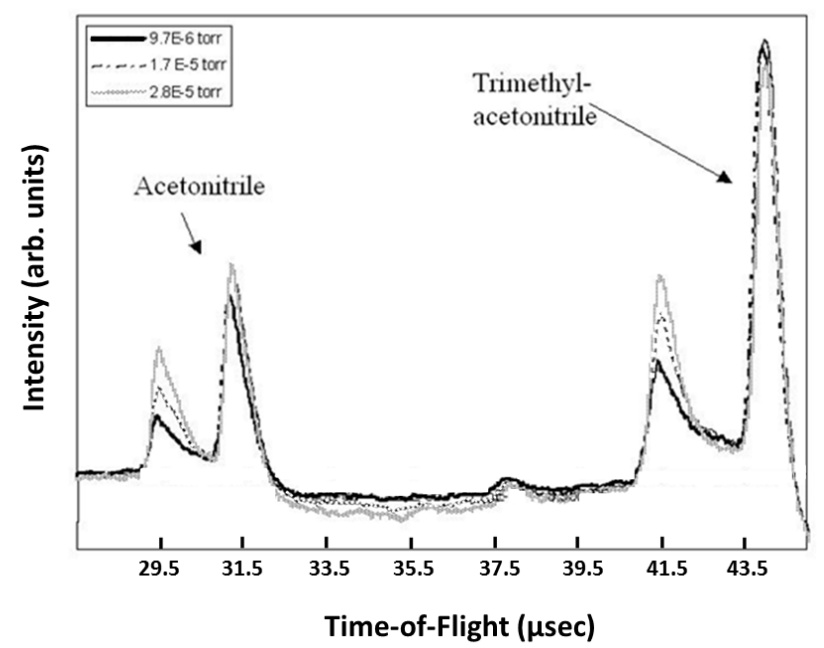

Figure 1. Time-of Flight mass spectra showing dipole bound anions of acetonitrile and trimethylacetonitrile. The corresponding peaks of slightly shorter time are the neutral signals formed from autodetachment of the ions while in the flight tube. The overlying spectra represent varying pressures in the interaction region during the RET process.

Fig. 2 shows the ion and neutral signals from production of dipole-bound anions of acetone and cyclobutanone. As with the nitriles, the dependence on the pressure in the interaction region is clear. In this case we see a noticeable dip in the ion signal of cyclobutanone at higher pressure, possibly due to collisional detachment as well as the autodetachment from rotationally excited dipole-bound anions. Again the relative amount of neutrals observed is higher for acetone than for cyclobutanone even though acetone has a slightly higher electron affinity. However, it does have a lower moment of inertia along the axes perpendicular to its dipole moment. Thus for both pairs of dipolar molecules under study, larger auto-detachment cross sections correspond to smaller moments of inertia along the axes in which the electron can not "keep up" with the rotation. 


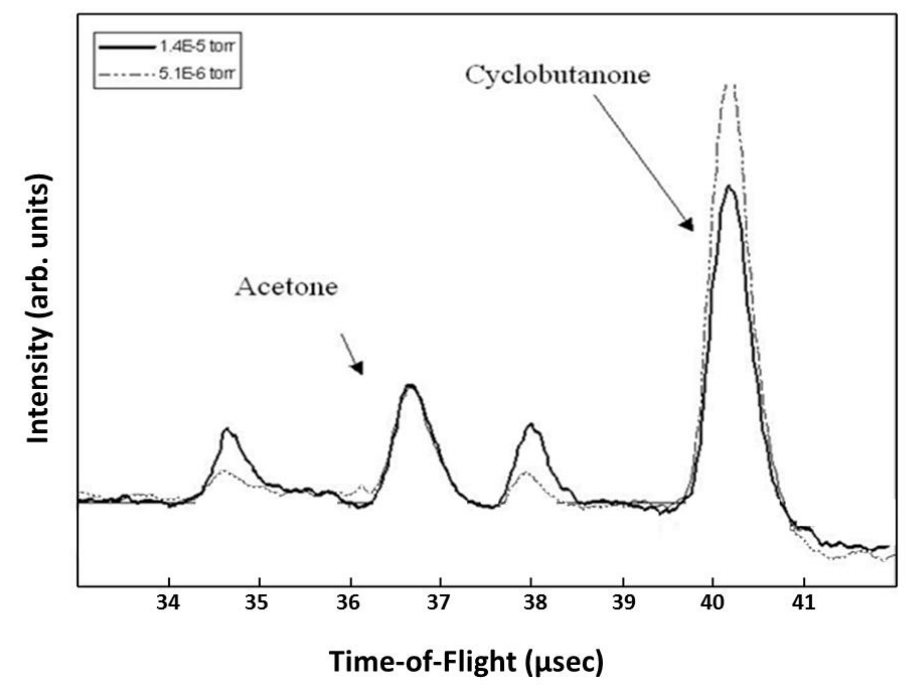

Figure 2. Time-of Flight mass spectra showing dipole bound anions of acetone and cyclobutanone. The corresponding peaks of slightly shorter time are the neutral signals formed from auto-detachment of the ions while in the flight tube.

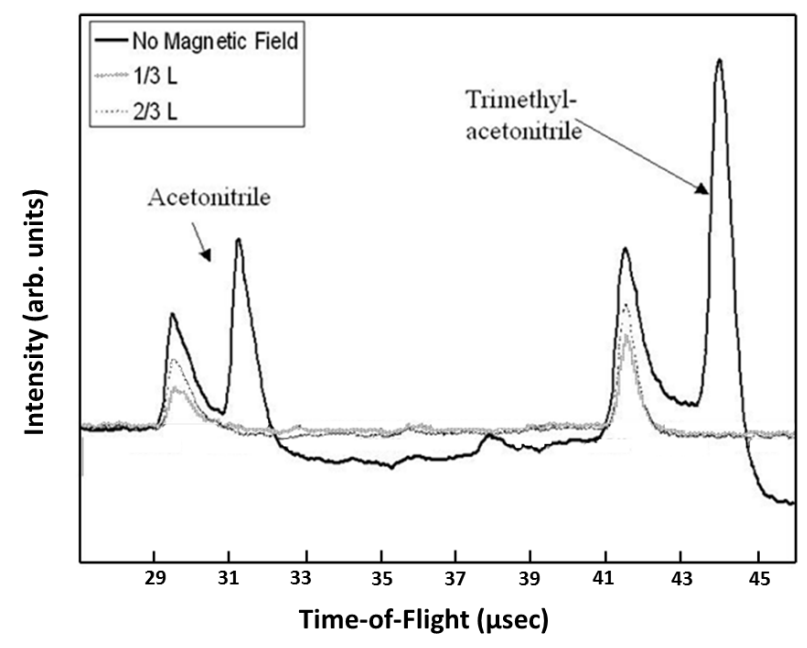

Figure 3. Time-of-flight spectra for the nitrile molecules, at an interaction region pressure of $2.8 \times 10^{-5}$ torr, showing the influence of a magnetic field orthogonal to the flight tube $1 / 3(1 / 3 \mathrm{~L})$ and $2 / 3(2 / 3 \mathrm{~L})$ of the way down the flight tube.

Important qualitative information on the relative auto-detachment rates of the rotationally excited dipole bound anions is found from considering the signal in the presence of a magnetic field perpendicular to the flight path. A permanent magnet was placed at approximately $1 / 3 \mathrm{~L}$ and $2 / 3 \mathrm{~L}$ the length (L) of the flight tube. Figure 3 show this data for the nitrile molecules for interaction region pressures $9.8 \times 10^{-6}$ torr. Placing the magnetic field anywhere along the flight path extinguishes the ion signal completely, as expected. Depending on the placement of the field, a percentage of the neutral signal is deflected as well, indicative of the auto-detachment not having yet taken place. One 
might be concerned about Lorentz stripping of the weakly bound electron producing neutral signal, potentially interfering with any temporal effects observed. If we consider the electron independently, as it is in a very diffuse orbital, a qualitative assessment of the Lorentz force applied is about $6 \mathrm{~N}$ under these experimental conditions. Comparatively, the force applied by the electric field during field dissociation measurements of acetone ${ }^{14}$ is on the order of $60,000 \mathrm{~N}$, leading us to believe the variation in neutral signal observed to be entirely due to auto-detachment taking place in the flight tube. We see that acetonitrile, (the lower mass peak) has a larger percentage of its neutral signal still carrying the excess charge at $1 / 3 \mathrm{~L}$ than that of trimethyl-acetonitrile, with comparable percentages at $2 / 3 \mathrm{~L}$. This could be interpreted as the acetonitrile anions being slightly longer lived, which is supported by their faintly larger binding energy. However other factors such as their collisional and blackbody photo-detachment cross sections must be taken into account before placing too much weight on this simple qualitative assessment. At larger pressures, a greater percentage of the neutral peaks remain undeflected at both field positions. This further supports the claim that increased rotational excitation is due to collisions with the background gas which lowers the respective lifetimes towards autodetachment.

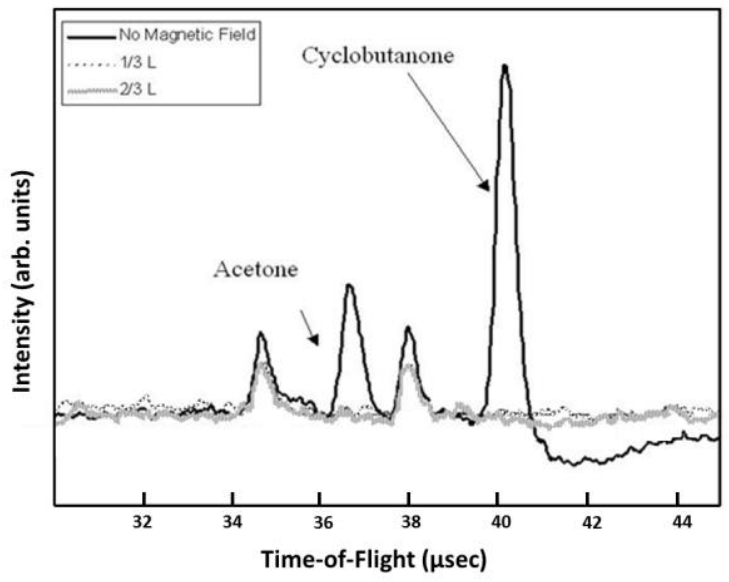

Figure 4. Time-of-flight spectra for the carbonyl molecules, at an interaction region pressure of $1.4 \times 10^{-5}$ Torr, showing the influence of a magnetic field orthogonal to the flight tube $1 / 3(1 / 3 \mathrm{~L})$ and $2 / 3(2 / 3 \mathrm{~L})$ of the way down the flight tube.

Figure 4 show this magnetic field data for the carbonyl containing anions with interaction region pressure of $1.4 \times 10^{-5}$ Torr. We see that both molecules exhibit the same amount of deflection at both field points. This means that no auto-detachment occurred in the middle $1 / 3$ of the flight tube. Most of the signal remains undeflected implying rapid auto-detachment, yet some deflection remaining at 2/3 L indicates auto-detachment taking place on a longer time scale as well. These two different auto-detachment rates 
may be explained by the contribution of blackbody photo-detachment, calculated to be on the order of 70 to $100 \mu \mathrm{sec}$, to the observed auto-detachment.

\section{Theoretical Observations}

As already noted, a non-Born Oppenheimer treatment of linear polar anions where rotational degrees of freedom are included in the dynamics, results in a very truncated set of bound rotationally excited states for molecules with dipole moments greater than the critical values for given systems. (The critical electric dipole moments depend on moments of inertia and rotational quantum numbers.) This property was verified in experimental measurements of the hydrogen cyanide anion spectrum ${ }^{19}$.

However, as recently shown by Garrett, ${ }^{27}$ the rotational spectra for polar symmetric top (ST) anions can be quite different from those of linear anions. For polar symmetric top rotors possessing rotational quantum number $J$ with projection $K$ about the symmetry axis, the rotor (and the dipolar vector) precess about the axis of quantization. The molecule and the dipole vector $\vec{\mu}$ are "oriented" when $K>0$. For $K=0$ the system is well treated as a linear dipole rotor. Thus a time-averaged dipole field is defined with dipole moment equivalent to a fixed dipole whose dipole moment $\bar{\mu}$ is the value projected onto and aligned along the processional axis. The time scale of the precession is very fast as compared to the motion of critically bound electron, thus the time-averaged value can be properly used. (The reverse Born-Oppenheimer approximation of Garrett ${ }^{20}$.) This reduces the problem of a ST rotor with dipole moment $\mu$ to that of a fixed dipole with the projected dipole moment.

$$
\bar{\mu}=\mu K / \sqrt{J(J+1)}
$$

With this result the problem is reduced to a critically bound electron in the field of a stationary dipole. Then on the basis of solutions for the fixed dipole problem, a new set of critical dipole moments can be defined as follows. When $J>0$ and $K>0$ the ST dipole rotor can support an infinite set of excited states for the given $J, K$ combination when this projected dipole moment exceeds the critical value

$$
\mu_{c}^{\prime}=0.639 \sqrt{J(J+1)} / K e a_{0}
$$

This critical moment is greater than that for a linear static dipole, where the critical moment $\mu_{c}=0.639 e a_{0}$, but it applies to the dynamic system and it depends only on $J$ and $K$, independent of the magnitudes of the inertial moments ${ }^{27}$. Of course the most probable values of $J$ and $K$ do depend on the temperature and the inertial moments, so the new critical moments are indirectly influenced thereby. (For the case $K=0$ the spectrum is like that of the linear rotor.)

The present experimental study was not designed to probe the recently discussed properties of polar ST anions since the experiments preceded Ref. 27. However, the data can be examined in the context of the theory for consistency, and for any additional insight into their interpretation. We will consider the data for the ST pair, acetonitrile and 
trimethyl-acetonitrile in the light of their excited state spectra and the implications for the observations.

We note that in seeded nozzle-jet expansions all rotations perpendicular to the symmetry axis are quenched far more effectively than rotations about the symmetry axis, especially for polar symmetric tops. For discussion purposes, we will assume that rotations about the axis of symmetry are not quenched in the expansion, so the values of the $K$ quantum numbers are unchanged from their initial distribution at room temperature. For purposes of the present comparisons, we assume that the distribution of total angular momentum $J$ is reduced in the expansions to that at an effective temperature $T$. However, the actual terminal temperature is not known in the experiments.

Assuming that the internal rotations are unquenched in the expansion we note that the distribution of the $K$ quantum numbers for acetonitrile at 300 Kelvin has maximum probability at $K=9$. The distribution is spread over a range where the probability has dropped to $10 \%$ of this maximum at $K=23$. For trimethyl-acetonitrile, with its much larger inertial moments, the axial rotational distribution peaks to a maximum at $K=49$ and is spread widely with the probability dropping to $10 \%$ of its maximum at $K=135$.

As noted, terminal temperature of the expanding gas is not well defined. Thus the discussion is limited to qualitative analysis of the comparisons between the two ST anions. For the purpose of assessing infinite critical binding for the two species, we assume two temperatures, $\mathrm{T}=100$ and 200 Kelvin and examine $\mu_{c}^{\prime}$ under the selected conditions. Thus for acetonitrile at $100 \mathrm{~K}$ the angular momentum distribution peaks at $J=20$. At this peak value $\mu_{c}^{\prime}=1.45 e a_{0}$. At a higher angular momentum where the distribution in $J$ has dropped to $10 \%$ of its peak value, $(\mathrm{J}=59)$, the required moment for infinite bound states is $\mu_{c}^{\prime}=4.26 e a_{0}$. For an assumed temperature of 150 Kelvin, the most probable $J=24$ and $\mu_{c}^{\prime}=1.74 e a_{0}$. These values are tabulated in table 2 . More generally, for acetonitrile with $K=9$ the excited state spectrum is infinite for $J \leq 21$ and finite for $J \geq 22$.

Now note the interesting circumstance that the probability peak for $J$ at 100Kelvin lies just below the transition from finite to infinite states which occurs at the $\mathrm{J}=21-22$ interval, and the most probable value at 150 Kelvin occurs just above the magic interval. Thus almost all molecules below the most probable total angular momentum support infinite bound states, while those that exceed the peak $\mathbf{J}$ support a finite set of bound states and resonances. This is approximately true for each assumed temperature.

Acetonitrile $\left(\mu=1.54 e a_{0}, K=9\right)$

\begin{tabular}{|l|l|l|l|l|l|}
\hline$T$ Kelvin & $J$ (at peak) & $\mu_{c}^{\prime} e a_{0}$ & $\begin{array}{l}J(\text { at } \\
10 \%)\end{array}$ & $\mu_{c}^{\prime} e a_{0}$ \\
\hline 100 & 20 & 1.45 & 59 & 4.26 \\
\hline 150 & 24 & 1.74 & 68 & 4.83 \\
\hline
\end{tabular}


Table 2. Dipole moments required for infinite critical binding for a rotor with acetonitrile physical parameters. Rotational quantum number $K$ at most probable room temperature value, $\mathrm{K}=9$.

Trimethyl-acetonitrile $\left(\mu=1.55 e a_{0}, K=49\right)$

\begin{tabular}{|l|l|l|l|l|l|}
\hline$T$ Kelvin & $J$ (at peak) & $\mu_{c}^{\prime} e a_{0}$ & $\begin{array}{l}J(\text { at } \\
10 \%)\end{array}$ & $\mu_{c}^{\prime} e a_{0}$ \\
\hline 100 & 49 & .645 & 133 & 1.73 \\
\hline 150 & 52 & .684 & 162 & 2.11 \\
\hline
\end{tabular}

Table 3. Dipole moments required for infinite critical binding for a rotor with trimethylacetonitrile physical parameters. Rotational quantum number $K$ at most probable room temperature value, $\mathrm{K}=49$.

For trimethyl-acetonitrile where most probable $K=49$, the most probable $J$ peaks at $J=49$ at $100 \mathrm{Kelvin}$ and at $J=52$ for $150 \mathrm{Kelvin}$. The corresponding values of $\mu_{c}^{\prime}$ are

listed in table 3. More generally, for this anion the spectrum is infinite for $J \leq 119$ and finite for $J \geq 120$. Thus, for trimethyl-acetonitrile almost all molecules support an infinite spectrum at $\mathrm{T}=100 \mathrm{~K}$ and the vast majority behave similarly even at $150 \mathrm{~K}$. On the other hand most of the distributions for acetonitrile lie above the cut-off for infinite states at $\mathrm{J}=21$.

Now we can qualitatively analyze the present experimental results for the ST acetonitrile and trimethyl-acetonitrile anions on the basis of the spectral features predicted here. The anions are formed through an electron transfer to a "state" of the ST. For neutrals in initial states $J, K$ that lie below the critical $\mu_{c}^{\prime}$ all electron transfers go into one of a limited set of bound states or of resonant (unstable) excited states. This is true for the majority of the acetonitrile species. On the other hand, for ST systems in a similar initial $J, K$ configuration but with a dipole moment above $\mu_{c}^{\prime}$ an electron can be transferred into an infinite number of bound states. This is true for the vast majority of the trimethylacetonitrile species. These are not subject to auto-detachment, but still yield to collisional or photo-detachment.

Auto-detachment behavior is much more complicated for ST than for linear polar rotors. However the properties are completely consistent with the comparative data on the two subject anions. The survival rate for the distribution of trimethyl-acetonitrile anions is greater than those of acetonitrile since a large fraction of the former do not auto-detach at all (though all are subject to photo-detachment), whereas in the former, most of the distribution will auto-detach. Although trimethyl acetonitrile is not a true ST, it is well approximated by one in the present treatment. The small deviations from ST behavior would have a small effect on the rotational spectra, however this would have no impact on the behavior relative to acetonitrile observed here. 
The additional set, acetone and cyclobutanone are not of ST form, though the cyclobutanone is closer to the ST form than acetone. However, the data for these two are qualitatively consistent with a true ST pair. That is to say, cyclobutanone would have a larger number of stable anion states, ie. those with $\mu^{\prime} \geq \mu_{c}^{\prime}$, due to the larger moment of inertia, which is consistent with what was observed.

\section{Conclusions}

The bound state properties of dipolar anions have been subjects of interest for a number of years. Many examples of dipole bound anions have been examined both theoretically and experimentally. However, experimental data on the actual spectra of real molecules, that is ground state plus excited states, has been quiet limited. Experimental studies of $\mathrm{HCN}^{-}$established that only a limited set of bound states exist, in agreement with theory ${ }^{19}$. However, the additional spectral properties of ST polar anions that can be inferred from recent work $^{27}$, have as yet not been examined experimentally. In the present study we have shown that the lifetimes of dipole bound anions are heavily influenced by rotational excitation and the properties have been shown to be consistent with predicted features of the ST bound state spectra. Rotational excitation imparted by collisions with background gas in the interaction region, or even through the RET process itself, allows for overalldetachment on a much smaller timescale than that calculated for blackbody photodetachment. Here molecules with similar dipole moments and electron affinities, and thus similar predicted blackbody photo-detachment rates, show noticeably different detachment rates dependent on the respective moments of inertia orthogonal to the dipole moment. The predicted behavior is more complicated for ST anions than for linear systems, and is in agreement with that observed here experimentally. A varied experimental approach with a longer region of deceleration of the ion signal may allow for a more quantified exploration of the observed lifetimes through analysis of the neutral peak shape, but would require significant modification to the present apparatus. However, qualitative analysis of the data illustrates the importance of critical binding properties and rotational auto-detachment in assessing the stability and lifetime of dipole bound anions in ST and near ST molecules.

\section{Acknowledgments}

We are grateful for the support of NSF during the course of this work.

\section{References:}

1. E. Fermi and E. Teller, Phys. Rev. 72, 406 (1947).

2. A. S. Wightman, Phys. Rev. 77, 521(1950).

3. J. M. Levy-Leblond, Phys. Rev. 153, 1 (1967).

4. M. H. Mittleman and V. P. Myerscough, Phys. Letters 23, 545 (1966) .

5. J. E. Turner and K. Fox, Phys. Letters 23, 547 (1966) .

6. W. B. Brown and R. E. Roberts, J. Chem. Phys. 46, 2006 (1967).

7. R. F. Wallis, R. Herman, and H. W. Milnes, J. Mol. Spectry. 4, 51 (1960). 
8. J. E. Turner, V. E. Anderson, and K. Fox, Phys. Rev. 174, 81 (1968).

9. O. H. Crawford, Proc. Phys. Soc. (London) 91, 279 (1967).

10. W.R. Garrett, Chem. Phys. Lett. 5, 393 (1970).

11. O.H. Crawford and W.R. Garrett, J. Chem. Phys. 66, 4968(1977).

12. C. Desfrancois, H. Abdoul-Carmine, J.P. Schermann, Int. J. Mod. Phys. B 10:1339(1996)

13. R.N. Compton, N.I. Hammer, Advances in gas-phase ion chemistry, Volume 4, p. 257-305, Amsterdam:Elsevier Science Ed. N. Adams and L. Babcock.

14. N.I. Hammer, K. Diri, K.D. Jordan, C. Desfrancois, and R.N. Compton, J. Chem. Phys. 119, 3650(2003).

15. L. Suess, Y. Liu, P. Parthasarathy and F.B. Dunning, J. Chem. Phys. 119, 12890(2003).

16. L. Suess, Y. Liu, P. Parthasarathy and F.B. Dunning, Chem. Phys. Lett. 376, 376(2003).

17. V.E. Chernov, A.V. Danilyan, A.V. Dolglikh, F.B. Dunning and B.A. Zon, Chem. Phy. Lett. 426, 30(2006).

18. W.R. Garrett, Phys. Rev. A 3,961 (1971).

19. S. Ard, W.R. Garrett, R.N. Compton, L. Adamowicz, and S.G. Stepanian, Chem. Phys. Lett. 473, 223 (2009).

20. W.R. Garrett, J. Chem. Phys. 133, 224103 (2010).

21. K. Fossez, N. Michel, W. Nazarewicz, and M. Ploszajczak, Phys. Rev. A 87, 042515 (2013).

22. W.R. Garrett, Phys. Rev. A 11,509 (1975).

23. D.C. Clary, J. Phys. Chem. 92, 3171 (1988).

24. J. Simons, J. Phys. Chem. 91, 6858 (1989).

25. E.A. Brinkman, S. Berger, J. Marks, and J.I. Brauman, J. Chem. Phys. 99, 7586 (1993).

26. A.S. Mullins, K.K. Murray, C.P. Schultz, W.C. Lineberger, J. Phys. Chem. 97, 1028 (1993).

27. W.R. Garrett, J. Chem. Phys. 141, 164318 (2014).

28. Scientific Instrument Services, Inc. 1027 Old York Road. Ringoes, NJ 085511054 
Graphical Abstract

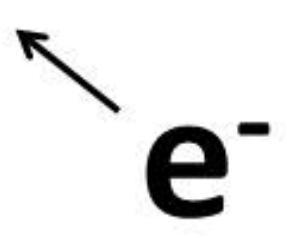

$$
\mu_{c}^{\prime}=0.639 \sqrt{J(J+1)} / K
$$

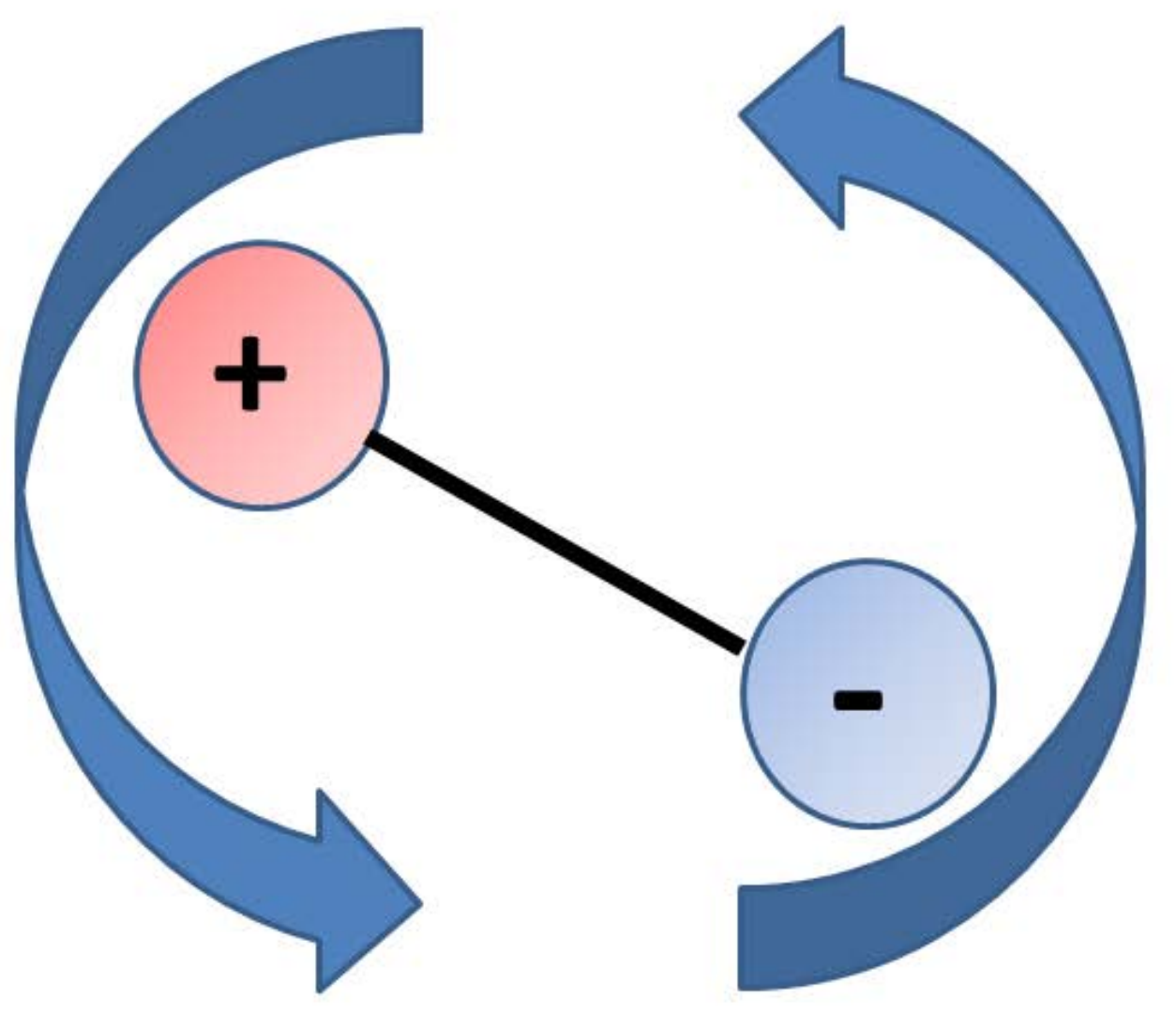

